\title{
Investigación en la Universidad Nacional de Asunción
}

\section{María Teresa Rosas de Maidana}

\author{
Facultad Politécnica-UNA
}

Recibido: 20 de octubre de 2014

Aceptado: 12 de noviembre de 2014

\section{Resumen}

El presente artículo tiene como objetivo analizar algunas de las razones por las cuales debe hacerse investigación científica en las universidades públicas en Paraguay. Se resalta así la importancia de investigar para la formación de recursos humanos, no sólo por la alta preparación que genera, sino también porque estimula la curiosidad, la innovación y la creatividad. Se cimienta de este modo los fundamentos para el desarrollo integral del país. En este trabajo se acentúa la necesidad de lograr una mayor articulación entre los organismos de ciencia y tecnología y las universidades sobre la plataforma de la investigación aplicada y la tecnología. Se busca de este modo dar respuesta a uno de los tantos problemas nacionales como es la cooperación interinstitucional como ruta para la transferencia de la información generada para el logro de un país mejor.

Como resultado, se enfatiza en la influencia del quehacer investigativo en la cultura actual, como también la importancia para la valoración de las mismas universidades.

\section{Pallabras claves}

Investigación Científica, Tecnología-Investigaciones, Ciencia y Tecnología

\section{Research at Asuncion National Univeristy}

\section{Abstract}

This article aims to analyze some of the reasons that scientific research should be done in public universities in Paraguay. The importance of research to the development of human resources and highlights not only preparing generating high but also because it stimulates curiosity, innovation and creativity. It thus builds the foundation for the development of the country. In this work the need for greater coordination between the science and technology agencies and universities on the platform of applied research and technology is emphasized. It thus seeks to answer one of the many national problems such as inter-agency cooperation as a route for the transfer of information generated for achieving a better country.

As a result, it emphasizes the influence of research activities in today's culture, as well as the importance for the assessment of the universities themselves

http://dx.doi.org/10.5209/rev CDMU.2014.v25.47476 


\section{Keywords}

Scientific Research, Technology-Research, Science and Technology

\section{INTRODUCCIÓN}

Una de las funciones primordiales de la universidad es propiciar la creación de nuevos conocimientos a través de la investigación científica, tecnológica, humanística y social. Esta función se ha convertido en el centro de la evaluación de las universidades en muchos países del mundo y en Paraguay.

Hoy la fusión universidad e investigación básica, que ha predominado por muchos años, comienza a ser cuestionada por los elevados costos y porque para muchos, ésta no aporta resultados "útiles", "rentables", capaces de producir recursos para las universidades. La constante presión sobre los investigadores para que realicen investigación aplicada ha intervenido para que muchos científicos de elevado nivel se dediquen a actividades que responden sólo a la necesidad de una investigación rentable y a la producción de ingresos en trabajos rutinarios, la mayor parte de las veces relacionadas con la prestación de servicios (Mayz, 1997).

La creación de la Secretaria Nacional de Ciencias y Tecnología en abril de 2012, es un gran paso.

Esta Secretaría tiene a su cargo grandes desafíos dispuestos en 4 pilares principales: Infraestructura, Gobierno Electrónico, Desarrollo Económico, Capacitación y Concienciación. De este modo, se busca transformar al Paraguay en un país más confiable y transparente, proporcionando servicios digitales de calidad y creando así condiciones para lograr un desarrollo económico sustentable y sostenible.(Portal PY 2014)

Es innegable que el camino de la ciencia en Paraguay tiene terreno que recorrer. Por ende se hace una necesidad acentuar en este artículo el derecho a investigar sin la eterna presión de hacer ciencia "útil". Y valdría la pena preguntarse ¿llegará el día en que en Paraguay se reconozca el papel de la ciencia y a los científicos como creadores de conocimientos?, ¿llegará el día de hacer ciencia sin apellidos, de crear con libertad, de valorizar el conocimiento en la expresión más genérica y con el único compromiso de que sea ciencia de calidad?. Corresponde a los investigadores luchar por alcanzar el reconocimiento necesario a la labor investigativa y valuar las preguntas.

Es común cuestionar la importancia de la investigación científica básica, muchas veces de un elevado nivel que busca satisfacer las necesidades de los países en vías de desarrollo. Es preciso, señalar la relevancia de las investigaciones. No se trata de negar que una investigación cierta conduce a solucionar problemas económicos y sociales. Si es útil, pero él no aceptar el desconocimiento respecto al estudio realizado, da lugar a un mal entender de la acción, debe reconocerse que no es ventajoso de manera inmediata a la ciudadanía toda, pero en forma paulatina va adquiriendo su valor en el desarrollo país.

\section{La învestigación científica en la universidad}

Es ventajoso inquirir, si los profesores de las universidades realizan investigación científica acorde con lo que pudiéramos denominar una investigación conforme a necesidad. La respuesta parece ser negativa, puesto que la mayoría de los docentes universitarios no investigan. 
En Latinoamérica, con pocas excepciones, las universidades dedican sus esfuerzos a la docencia. La creación excesiva de universidades privadas en países como en Paraguay, Perú Chile y Bolivia han aumentado el número de universidades de "tiza y pizarrón", universidades que no desarrollan investigación, que sólo transmiten conocimientos. como lo señalan Lemansson y Chiappe (1999),

Pero, regresando al título y al propósito del presente artículo valdría la pena preguntarse: ¿para qué hacer investigación científica en las universidades?

A continuación una serie de motivos que la justifican.

\section{La investigación científica y la formación de recursos humanos. El postgrado}

La investigación es la que estimula el pensamiento crítico y la creatividad tanto en los docentes como en los alumnos. Es por medio de la investigación que el proceso de aprendizaje se vitaliza y se combate la memorización que tanto se ha contribuido a formar profesionales pasivos, poco innovadores, con escasa inquisición e iniciativa personal. Se puede señalar entonces que el perfil general del docente contrasta considerablemente con el que la Ley General de Educación y varios documentos establecen, en forma especial, el grado de criticidad y creatividad que debería caracterizarlo, en Paraguay. Por supuesto, mientras se forme este tipo de profesionales, habrá siempre una brecha que dejará a los países subdesarrollados cada vez más atrás de los países desarrollados. Acotando que el sistema educativo paraguayo sigue siendo inadecuado ya que condena a la perpetuación del subdesarrollo y a la marginalidad intelectual y científica.

El objetivo que justifica la necesidad de investigar en las universidades es la de preparar individuos con habilidades y conocimientos nuevos que tengan una formación académica adecuada para el desafío de un mundo urgido en desarrollo técnico-científico. Esto es así porque la sociedad requiere capital humano para resolver sus problemas más inmediatos; contribuir a acrecentar ese capital es una de las misiones más importantes de las universidades.

La herramienta fundamental para lograrlo es la ciencia; siendo de radical importancia cultivarla. El insumo más valioso hoy en día es el conocimiento y no resulta arriesgado decir que es la única inversión cierta.

Es significativo recordar que sólo mediante la investigación se podrá formar el recurso humano, a nivel de especialistas, con la alta calidad que el país necesita. Del mismo modo se debe reconocer que la investigación es de un valor trascendental en los estudios de postgrado; no es posible tener egresados de alto nivel si no se investiga.

Respecto a lo expuesto, Pérez-García (1997) en su material se plantea una serie de preguntas, de las cuales se destacan dos.

La primera es si se está inculcando de verdad el espíritu científico a los jóvenes de nuestras universidades.

La segunda, si es el ambiente de las universidades verdaderamente participativo y desafiante para el intelecto del estudiante.

La respuesta se obtendrá en el desafío de la reforma de la educación superior y el cultivo y difusión de la cultura científica. 


\section{La cultura, la ciencia y la investigación científica.}

Todo país tiene la obligación de desarrollar su propia cultura. Un país culto y civilizado presume de sus orquestas sinfónicas, de sus biblioteca y museos, de sus escritores y personajes que marcaron épocas en beneficio país. Pero en pocas ocasiones, se considera el valor de los científicos. No se percatan de que la ciencia se está convirtiendo en el núcleo de la cultura moderna. Tampoco suele apreciarse que para filosofar con sentido y rigor en el siglo XXI se precisa estar en conocimiento de las grandes conquistas y de los grandes problemas de la ciencia, para así adoptar una actividad científica ante los problemas filosóficos (Bunge, 1981).

La ciencia no sólo es el cimiento de la tecnología que está dando una nueva fisonomía a la cultura material, sino que absorbe disciplinas que otrora fueron artísticas y filosóficas: ayer, la antropología, la psicología y la economía; hoy, la sociología y la historia, mañana, quizá, la estética y la ética (Bunge, 1981). Además, la concepción del mundo del hombre contemporáneo se funda, en medida creciente, sobre los resultados de la ciencia: el dato reemplaza al mito, la teoría a la fantasía, la predicción a la profecía. La cultura social y la personal se tornan, en suma, cada vez más científicas. Hace un siglo, quien ignoraba La llíada era tildado de ignorante; hoy lo es, con igual justicia, quien ignora los rudimentos de la física, de la biología, de la economía y de las ciencias formales (Bunge, 1981).

\section{La învestigación científica y el desarrollo tecnológíco}

La investigación básica que predomina en las universidades del mundo es necesaria en los países subdesarrollados tal vez incluso en mayor medida que en los países avanzados, porque es allí de donde sale la inspiración creativa para lo tecnológico. Contrariamente a lo que piensan los que están ajenos al devenir de la ciencia, el progreso de la investigación aplicada o tecnológica no se logra distrayendo recursos de la ciencia básica, sino reforzándola.

\section{La investigación científica y la información}

No se puede obviar que, en el mundo actual, el verdadero ejercicio de la libertad y la soberanía están en el conocimiento. El vahído de una ciencia y una tecnología propia contribuiría a un empobrecimiento social con consecuencias insospechadas y a una mayor dependencia de los países desarrollados. En el futuro, las sociedades avanzarán sobre la base del acceso al conocimiento (Pérez et al., 1999).

La información es poder. Pero quiénes son realmente los que pueden establecer cuál es la tecnología central en un paquete tecnológico. Lógicamente, es el investigador quien detecta ese tipo de tecnología. Carbonell (1979) relata una interesante experiencia en el establecimiento de una fábrica de bioproteínas con la British Petroleum. Se estudió con mucho cuidado la parte de la tecnología que se estaba vendiendo y la conclusión importante fue que la mayor parte de la millonaria inversión se centraba en un solo punto importante: una cepa de hongos que se debía usar. De esto soslayamos el análisis cierto de las necesidades para la investigación.

\section{La investigación científica para tener un mejor país}

Sin lugar a dudas, el principal problema que enfrenta Paraguay es la pobreza. Todos los demás, incluso el de la inseguridad personal, son consecuencias de ésta. La causa principal de la pobreza es la ignorancia. Es evidente que la democracia y los derechos a la educación consagrados en la Constitución Nacional, no se 
han traducido en el desarrollo de una mejor sociedad que dependa en forma exclusiva de las riquezas naturales del país. Se necesita de la ciencia para disminuir los límites de la ignorancia y aumentar la capacidad para resolver los problemas. En el concepto ciencia también está implícito el de tecnología, pues aunque esta tiene sus predicamentos diferenciales, está íntimamente asociada a la ciencia propiamente dicha.

Pérez-García (1997), por su parte, señala que es común pensar que los éxitos de un país se deben sólo al buen manejo de las políticas macroeconómicas, a decisiones empresariales adecuadas o a oportunidades del mercado nacional e internacional. Se obvia que las condiciones para que este escenario sea posible son el conocimiento de las tecnologías pertinentes y el de un personal técnico bien capacitado. Es decir, es fundamental disponer de una capacidad científica y tecnológica actualizada que permita desarrollar y solucionar con las mejores tecnologías disponibles, preparar a los profesionales necesarios y que tengan un conocimiento pleno de los recursos y posibilidades para el I+D.

El Paraguay es uno de los países sudamericanos que presenta un panorama menos denso respecto a la investigación científica y con escaso producto de difusión universal. Ha habido aislados inmigrantes científicos de valor como Rengger, Bondpland, Bertoni, Hassler y otros, pero la ciencia institucional entendiéndola como la que se practica no como entretenimiento, sino como un trabajo en una institución, típicamente la universidad, es muy incipiente según lo expresa el presidente de la Sociedad Científica del Paraguay, Manuel Gill Morlis (2014). Hay dos esfuerzos específicos recientes en el país, uno es un programa de sueldos decentes para profesores investigadores a tiempo completo en la Universidad Nacional de Asunción y otro es el creado el Programa Nacional de Incentivo a Investigadores (Pronii) del Consejo Nacional de Ciencia y Tecnología (CONACYT) y la colaboración cercana de los ministros de Hacienda y Salud Pública.

Manifiesto es que la Universidad Nacional de Asunción, creada en 1889 invierte con recursos propios del rectorado en un promedio de 51 investigaciones al año, según la dirección General de Investigación Científica y Tecnológica (DGICT).

Las unidades académicas más favorecidas son:

- Ciencias agrarias con 83 investigaciones

- Ciencias Químicas con 69 trabajos

- Politécnica con 65 proyectos

- Ciencias Exactas y Naturales con 57 trabajos

- Ciencias Económicas con 44 proyectos

- Ingeniería con 42 trabajos

- Ciencias Médicas con 40 trabajos

- Ciencias Veterinarias con 28 proyectos

- Arquitectura, Diseño y Arte con25 proyectos a igual que Filosofía y Odontología. 
El instituto de Investigaciones en Ciencias de la Salud con 24 trabajos, el Centro Multidisciplinario de Investigaciones Tecnológicas con 19 proyectos, Derecho y Ciencias Sociales con 14 investigaciones y el Andrés Barbero con 4 proyectos.

Se destacan los proyectos: Desarrollo de pasta dental con revelador de placa bacteriana, con patente en trámite, Estudio del contenido de aflotoxinas y hongos aflotoxigénicos en yerba mate comercial paraguaya, Determinación de metales pesados en aguas del río Paraguay, entre otros mencionó el Ing. César Cardozo, director general del DGICT en entrevista en un medio televisivo.

La UNA cuenta con la figura del Docente Investigador de Tiempo Completo (Dicode) desde el año 2007, con 62 profesionales calificados en esta área y 581 investigadores de tiempo parcial. En la categorización de niveles (4) dado por el CONACYT a los 386 acreditados 72\% guardan relación con la UNA (DGICT,2014).

Desde 1993, surgen las Jornadas de Jóvenes Investigadores, que se desarrolla anualmente de manera ininterrumpida. En la misma, acuden un promedio de 800 investigadores de la región y 300 docentes de todas las Universidades miembros. En el año 2007, la Universidad Nacional de Asunción (UNA) fue anfitriona de las "XV Jornadas de Jóvenes Investigadores", en la ocasión, acudieron cientos de investigadores de todos los países acompañados por sus respectivos docentes. En aquella oportunidad, la UNA demostró contar con la infraestructura y el recurso humano necesario para llevar adelante tan magno evento. El año 2007 también marcó el inicio de una postura decidida por parte de las autoridades y directivos de la UNA para implementar el programa Jóvenes Investigadores. Desde entonces, de manera sostenida, la UNA participa con el mayor número de trabajos posibles en cada evento a nivel regional. Asimismo, se está trabajado en cooperación con docentes y estudiantes Por otro lado, la comunicación fluida y precisa de las ideas, resultados y conclusiones de los trabajos de investigación, han motivado una serie de talleres de expresión oral y manejo de estrategias de comunicación con docentes.

El Rectorado de la UNA, por su parte, ha brindado todo su apoyo poniendo a disposición de los investigadores jóvenes toda la logística para el traslado (buses) correspondiente y en condiciones inmejorables. Asimismo, los Decanos de las Facultades y los directivos y responsables de los centros e institutos han apoyado la investigación otorgando a cada investigador seleccionado/a la ayuda necesaria para la estadía y la manutención. Este esfuerzo mancomunado da firme apoyo a los jóvenes investigadores, transformado de este modo la dirección que afianza los lazos de comunicación entre los investigadores jóvenes y entre los docentes de la región.

Del mismo modo, la UNA, con satisfacción y orgullo, ha sido reconocida con 10 menciones por la calidad y rigurosidad de los trabajos de investigación presentados. Cabe destacar, cada año en el mes de septiembre, en el marco de la Exposición Tecnológica y Científica (ETyC) de la Facultad Politécnica, son seleccionados los trabajos para los eventos internacionales que son expuestos a todo público. Cabe mencionar que varios de los jóvenes participantes, hoy día usufructúan becas en universidades europeas de prestigio y renombre en el área de investigación El hecho de haber participado en las jornadas de Jóvenes Investigadores de la Asociación de Universidades Grupo Montevideo( AUGM) les ha proporcionado una ventaja adicional, tanto a 
la hora de acceder a becas como para concursar como ayudantes de cátedras. También los trabajos de investigación de los jóvenes de la UNA que participaron en los eventos internacionales, todos ellos fueron publicados en los libros de resúmenes. La revista Investigaciones y Estudios de la UNA, recoge los trabajos de las Jornadas de Jóvenes Investigadores y las publica.

Cada año, los jóvenes, gracias a esta interesante iniciativa, tienen la posibilidad de intercambiar ideas, proyectos e ir forjando realidades con sus pares de la región en pos de un Paraguay más próspero y con influencia en el MERCOSUR.

Tres son los trabajos de la Facultad Politécnica que fueron seleccionados para representar a la UNA en las XXII Jornadas AUGM que se llevarán a cabo del 29 de setiembre al 1 de octubre del 2014 en Valparaíso, Chile.

A continuación los títulos de las investigaciones, elegidas tras la presentación y competencia en las VIII Jornadas de Jóvenes Investigadores (JIII):

"Diseño de un modelo de programación matemática para la planificación de cosecha de hortalizas", de Jorge Luis Recalde Ramírez.

"Diseño de un sistema de radiodifusión sonora terrestre digital en Paraguay", de Rubén Darío Reinoso Escobar.

"Automatización del monitoreo de la conductividad eléctrica, humedad y temperatura del suelo agrícola" de Richard José González.

Estos trabajos fueron seleccionados, junto a los de otras Facultades de la UNA, de un total de 270 investigaciones presentadas y defendidas en las VIII JJII de la UNA, que se llevó a cabo el 15, 16 y 17 de julio del 2014 en el Campus de la UNA, San Lorenzo.

Generar, realizar y promover investigaciones científicas en el área de las ciencias de la salud, con el aporte de conocimientos y soluciones a los problemas relevantes del país, con la capacitación de recursos humanos y el desarrollo de servicios especializados, para contribuir a mejorar la salud de la comunidad" son las principales líneas de investigación del Instituto de Investigaciones en Ciencias de la Salud (IICS) relacionadas estas con los problemas prioritarios del país que son las enfermedades infecciosas (parasitarias, virales y bacterianas). Los datos resultantes de las investigaciones del IICS en estas áreas constituyen un aporte importante para el Ministerio de Salud Pública y Bienestar Social siendo un bien para los ciudadanos del Paraguay.

\section{Contribución}

Programa Nacional de Chagas. Chagas congénito

Programa Nacional de Leishmaniasis 
Programa Nacional de prevención y control del cáncer de cuello uterino

Programa Nacional de Hipotiroidismo congénito

Programa Nacional de Lucha contra el SIDA (en etapa inicial)

Vigilancia de la Salud

Distribución de aislados de variantes de VPH-16 en mujeres paraguayas con diferentes grados de lesión cervical (Premio Nacional de Ciencia 2014)

\section{Coordinación de actividades de docentes investigadores de tiempo completo y dedicación exclusiva} (DITCODE) del IICS.

La Coordinación de Investigación Científica coordina las actividades de los DITCODE del IICS ante la Dirección General de Investigación Científica y Tecnológica (DGICT) de la Universidad Nacional de Asunción (UNA) que opera desde 2007. Siendo la creación de DITCODE una iniciativa del Rectorado de la UNA como una nueva política de gestión de recursos humanos tendiente a la transformación académica y administrativa de la UNA, para alentar las actividades de creación, producción y transferencia de ciencia y tecnología desde el ámbito universitario hacia las realidades productivas de la comunidad, con su consecuente impacto social.

De allí se desprenden innumerables proyectos de investigación tales como:

Alumno: Gustavo Enrique Espínola Mena

Grupo de Investigación: Departamento de Ciencias Computacionales (DCC) Proyecto: Reversión dinámica de carriles vehiculares: una propuesta basada en sistemas distribuidos con Middleware Corba

Alumno: David Caballero Morilla y Federico Gavilán

Grupo de Investigación: Departamento de Sistemas de Potencia y Control (DSPyC)

Proyecto: Análisis y simulación de algoritmos de control de potencia activa y reactiva aplicados al modelo predictivo de un convertidor de potencia para aplicación en generación distribuida.

\section{En la FacultadVeterinaria}

Área extensión universitaria línea

Línea 1. Impacto social de las Ciencias Veterinarias

Área recursos faunísticos línea

Línea 1. Manejo, conservación y uso sostenible de animales silvestres.

Línea 2. Diagnóstico de enfermedades y terapéutica en especies silvestres y animales de compañía no convencionales.

Área medio natural 
Línea 1. Manejo de residuos y protección ambiental

Áreas animales de laboratorio

Línea 1. Manejo de especies de animales de laboratorio.

Línea 2. Diagnóstico de enfermedades de animales de laboratorio

Son entre tantas las que menciono en este material.

Menciones de honor del Concurso Nacional de Ciencia 2014:

Eficacias y seguridad de la utilización de dabigatan etexilato con doble terapia concomitante antiplaquetaria en la fibrilación auricular de Osmar Centurión.

Estudios geoquímicos y ambientales en la bahía de Asunción y el río Paraguay, de Juan F. Facetti.

Un servicio semántico basado en ontologías para equipamientos urbanos cooperativos de Derlis Gregor.

Geografía humana del Chaco paraguayo de Fabricio Vázquez.

\section{METODOLOGÍA}

El diseño metodológico de esta investigación es explorativa y descriptiva; es no experimental o ex postfacto, pues no considera la manipulación deliberada de los hechos ni de las variables, sino que supone un enfoque retrospectivo y variable de hechos similares ocurridos sin la intervención directa del investigador.

Para las técnicas de recopilación de datos se optó por la observación, entrevista no estructurada, así como la compilación de material documental referente.

Cotejado los datos con responsables de alto nivel jerárquico de los entes involucrados.

Constituida la muestra por las 12 universidades que conforman la UNA y su alcance temporal es desde 2010 hasta 2014.

\section{RESULTADOS}

Entre los resultados posibles está en las razones suficientes para el logro del fortalecimiento al grupo de investigadores de dedicación exclusiva, estimando el desarrollo de modelo a ser imitado por otras instituciones.

Y en la difusión de las investigaciones desarrolladas destacando que se hace necesaria una política proactiva en apoyo a los jóvenes investigadores para construir un nuevo país.

\section{CONCLUSIONES}

Existen suficientes razones para justificar la investigación científica en las universidades. Ésta debe ser la base de la educación, pues enseña a pensar, a comprender y a crear nueva información, conocimientos y tecnologías. Permite, además, formar profesionales con creatividad, dispuestos a innovar. 
Es importante resaltar que el conocimiento, la información y las tecnologías generados a través de la investigación juegan un gran papel en el desarrollo integral del país; por ende, debería existir una mayor articulación entre los organismos de ciencia y tecnología y las universidades.

Es innegable que la investigación permite vincular más el sistema educativo con los sistemas científicotecnológicos. Se hace necesario, entonces, la renovación de los objetivos y currículo de los pregrados que permitan sentar las bases que aseguren, posteriormente, una mayor integración entre la investigación y los estudios de postgrado.

La investigación aplicada y la tecnología, por su parte, permiten dar respuesta a problemas confrontados no sólo por el país, sino también por la comunidad latinoamericana, estos constituyen los pilares de la cultura actual.

Entendiendo de este modo que la investigación, la ciencia y la tecnología hacen la diferencia entre la cultura pasada y la contemporánea.

La investigación, en general, se ha convertido en una de las bases destacadas para evaluar las universidades. Debe estimularse y valorizarse, sin presionar a los investigadores a hacer ciencia "útil" o rentable logrando de esta manera la apropiación de la investigación científica por la ciudadanía.

\section{BIBLIOGRAFÍA}

Anales de la sociedad científica del Paraguay. Conferencias-2013. Asunción: Sociedad Científica del Paraguay,2014

Bunge, Mario (1981). La ciencia, su método y su filosofía. Buenos Aires, AG: Siglo Veinte, 1981. 99-145.

Carbonell, Jaime. (1979) El razonamiento basado en estudios de casos Actas de la Academia de Ciencias Naturales de Filadelfia 131,(1979), 104-130

¿Cómo promover el interés por la cultura científica? (2005). Santiago de Chile, OREALC/UNESCO (2005). www.oei.es/decade/1390035.pdf (2014-07-15)

Cubilla, Antonio. L. (2013) Estrategias para la inserción de las ciencias y tecnologías en el Paraguay. Asunción: Instituto de Patología e Investigación, 2013

Lemasson, Jean Pierre, Chiappe, Marta. (1999). La investigación universitaria en América Latina. Texas: IESALC/UNESCO, 1999.

Martínez, Eustaquio (2007) Investigación, universidad y desarrollo Revista Científica Politécnica, 3:3. (Marzo 2007) 75-76

Mayz Vallenilla, Ernesto.(1991). El ocaso de las universidades. Caracas: Monte Avila,1991.

Pérez, Julio. (1998). La investigación científica en Latinoamérica Interciencia, 23,(enero-febrero 1988) 316.

Pérez-García, Carlos. (1997). La investigación en la universidad y la formación integral del estudiante Postgrados, 3:1,(febrero 2013) 19-31. 
Programa Nacional de Incentivo a los Investigadores (2011). www.conacyt.gov.py/(2014-03-21)

Rectorado de la Universidad Nacional de Asunción. Dirección General d Investigación Científica y Tecnológica. Política científica del Paraguay2013. www.una.py (2014-08-2)

Silvero, José Manuel. (2012)Algunas consideraciones acerca de la investigación en la UNA. UNA Revista .7:1,( enero-abril 2012). 13-14.

La universidad y la investigación científica (2012) UNA Revista 7:1, (enero-abril 2012.) 3. 